\title{
A trace based method for realistic simulation
}

\author{
Philippe OWEZARSKI and Nicolas LARRIEU \\ LAAS-CNRS \\ 7, avenue du Colonel Roche \\ 31077 TOULOUSE Cedex 4 \\ FRANCE \\ e-mail: \{owe, nlarrieu\}@laas.fr
}

\begin{abstract}
This paper proposes a new approach for making simulations realistic. This approach is based on the principle of "trace driven simulation", i.e. using results of actual traffic traces analysis in order to reproduce the same experimental conditions in simulation. The main principle of the approach proposed in this paper deals with making simulation traffic sources replay under certain conditions - the actual traffic traces grabbed on actual networks. This paper describes the implementation of this approach in the NS simulator, and evaluates it by comparing the characteristics of traces obtained with our replay approach, with original data traces. The parameters that are considered for making the comparison are the usual traffic parameters as throughput, packet rate, etc., but also everything that is related to traffic dynamics, i.e. the second order statistical moments as autocorrelation of traffic or long range dependence.
\end{abstract}

\section{INTRODUCTION}

Simulation is an essential tool to provide a priori evaluation of a network. It evaluates if such a network can work, i.e. if it is free of bugs, checks its liveness, its integrity, and also makes some evaluations of its performances. Of course, simulating the Internet is not an easy task, especially because of its size (number of users and equipments), its complexity (number of protocols for instance), the behavior of its users, and of course, its fast evolution in terms of technology and usage. Because of all these features, it is very difficult to get some realistic simulations of the Internet, or at least of some small parts of it.

[PAX01] that addressed this issue said that it is not enough to focus on the problem of network topology for simulating the Internet, but it is also essential to solve the problem of traffic sources to make them reproduce as much as possible the actual traffic of the Internet in terms of applications and protocols as well as their behaviors. For that, [PAX01] recommends "trace driven simulation", meaning that traffic sources have to use some results of traffic traces analysis. In addition, it is said that because of the self regulation mechanisms of protocols (congestion control mechanisms of TCP for example), the "trace driven simulation" approach has to work at flow level, the simulation environment being in charge of "shaping" packets according to the simulated network topology and protocols. The NS network simulator, for example, has been designed in the framework of the VINT [FAL96] project for this purpose.

One of the main issue for simulating traffic sources is related to the absence of an always valid traffic model and most of the time that traffic sources used in simulations are very simple and follow constant, Poisson or Markov laws. Of course, such sources do not generate traffics with all the unregular properties of actual sources in the Internet.

However, it is not easy to build a traffic model including all the characteristics and features of actual traffics. And the accuracy and realism of simulations remain poor.

Therefore, this paper proposes a new approach for building traffic sources for simulators. The principle of this approach deals with making simulation traffic sources replay the actual flow $^{1}$ traces captured with monitoring equipments. It is a simple and efficient way to create in simulators traffic sources having all the real characteristics and features, and going through the need of a flow arrival model in the Internet, that is not available yet.

In addition, as the simulation environment (topology, network agents, etc.) are in charge of the shaping of packets, it is important to create it in order to make it generate traffic having the same characteristics as actual traffic. Previous work [PAR96] and [VER00] showed that Internet traffic characteristics as long range dependence (LRD), self-similarity, etc. are due to TCP (the main protocol used in the Internet) and its congestion control mechanisms. As congestion control mechanisms are based on pre-defined responses to losses, it seems that the main characteristics of actual traffic xto enforce in simulations is related to the loss process.

This paper describes the implementation of this approach in the NS simulator (section II), and evaluates it. This evaluation is made by comparing the real traffic that has been captured and the "same" traffic replayed in the simulator (section III). The parameters that are compared are of course the usual traffic parameters (throughput, packet rate, etc.), but also everything that is related to the traffic dynamics, in particular second order statistical moments as autocorrelation of traffic or LRD.

\section{A NEW TRACE BASED APPROACH FOR SIMULATION}

As a starting point for using our approach for traffic sources, a traffic trace that can give flow level information is required. This trace can be any kind of packet level traces (as TCPdump or DAG traces [CLE00]). Then, some tools have to be used

\footnotetext{
${ }^{1} \mathrm{~A}$ flow is classically defined as a set of packets having the same 5-tuple, i.e. same source and destination addresses, same source and destination port numbers, and same protocol.
} 
TABLE I

FLOW CLASSES USED TO REPLAY MONITORED TRACES

\begin{tabular}{|c|c|}
\hline Class & Loss rate of flow class (\%) \\
\hline \hline C0 & 0 \\
\hline C10 & $0-10$ \\
\hline C20 & $10-20$ \\
\hline C30 & $20-30$ \\
\hline C50 & $30-50$ \\
\hline C100 & $50-100$ \\
\hline
\end{tabular}

to extract the flows information. At the end of the process, it is required to have a flow file, having an entry for each flow. The parameters are:

- For a TCP flow, the beginning timestamp, the number of packets and their sizes. The NS replay agent is then going to start the flow at the right time and send all packets with their real size, the shaping of packets being realized by the sending and receiving TCP agents.

- For a UDP flow, the beginning timestamp, the number of packets, their sizes, as well as the time between every consecutive two packets. The principle to replay this flow is the same as for TCP, except that as UDP is not running any flow or congestion control algorithm, our UDP agent as to respect the sending times of all packets that depend on application and/or user.

A replay module has been developed for NS. The traces we used are traces from the WAND group (university of Waikato), captured in Auckland, NZ. They were captured on 10 Mbps Ethernet links at peak hours, and using DAG systems, thus guaranteeing a very accurate timestamping based on GPS clocks (accuracy is less than 2 microseconds).

For designing the topology of the network, the goal is to create the simplest topology able to reproduce the loss process in simulation. For that, we analyze each flow of the original trace and measure its loss ratio. The goal then is to reproduce thanks to the simulation topology the same loss ratio for each flow. In order to build a suited network topology, it is also necessary to extract from original traces other flow parameters:

- the loss rate experimented by each flow during their exchange on the network,

- the RTT (Round Trip Time) experimented by each flow,

- the average throughput got by each flow,

- the duration of each flow.

To limit the complexity of the simulation topology, and based on loss ratio analysis, we decided to define only six different loss classes of flows (cf. table I for details).

With the information extracted from original traces, we are able to deduce both bandwidth and queue length of each link of the simulation topology where the flow will be transmitted, depending on the loss class to which the flow belongs to.

The link bandwidth for class $i\left(B w_{C i}\right)$ is computed thanks to equation 1:

$$
B w_{C i}=\frac{\sum_{n=1}^{N_{\text {flow }}} d_{i} * T h_{i}}{d_{\text {trace }}}
$$

where:

- $N_{\text {flow }}$ is the number of flows of class $i$,

- $d_{i}$ is the duration of flow $i$,

- $T h_{i}$ is the average throughput of flow $i$,

- $d_{\text {trace }}$ is the trace duration.

Then, the queue length for class $i\left(Q L_{C i}\right)$ is deduced thanks to equation 2 :

$$
Q L_{C i}=B w_{C i} *\left(100-\text { rate }_{\text {loss }}\right)
$$

where:

- rate $_{\text {loss }}$ is the average loss rate (in \%) got by each flow of the class.

Finally, the experiment topology that will be used to replay the trace considered in this example is depicted on Figure 1 ( $R T T_{C i}$ is the average RTT of the whole flows belonging to the class $i$ ).

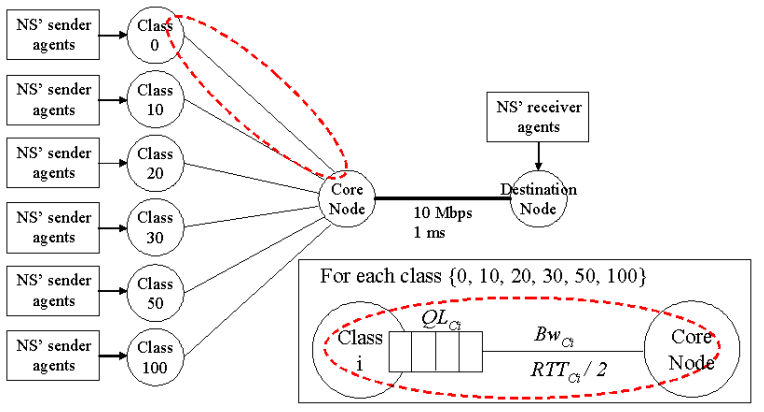

Fig. 1. Experiment topology

Traces have been monitored on a 10 Mbps Ethernet local network. That is why, in the experiment topology, the core link has a 10 Mbps capacity. The delay value for this link is $1 \mathrm{~ms}$ to avoid any influence on the sending of different class flows ${ }^{2}$. Indeed, the average RTT for each loss class has been computed and put on the different access links for the six loss classes.

In the next section, we present several experimental results to validate our replay approach. We have tested our replay method on a large number of traces, and we got very similar results with all of them. We are then just showing the results we got with one of them, comparing it with the replayed traffic. Recall that for the analysis we will mainly focus on traffic dynamics that are the most difficult parameters to reproduce and to control in simulation, and that are responsible of most the (performance) issues of the Internet. For example, it has been shown in the literature that the traffic characteristics causing the more issues are the highly oscillating nature of Internet traffic especially because of the dependence between the transmission of bytes and packets [PAX95], the dependence between losses or congestions [ZHA01], because of the heavy tailed flow size distribution that makes dependence phenomena propagate on very long range [CRO97]. Such features can be characterized with mathematical functions. In

\footnotetext{
${ }^{2}$ It should be 0 but it is impossible with NS.
} 


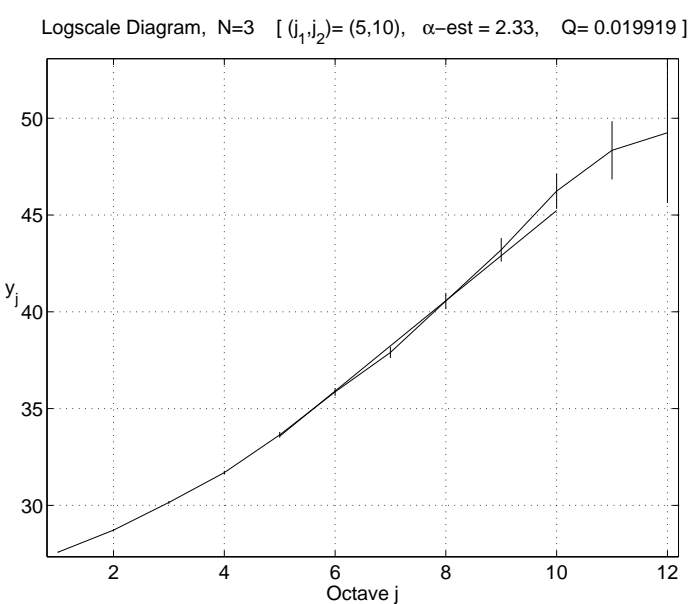

(b) Replay-based simulation (a) Original trace

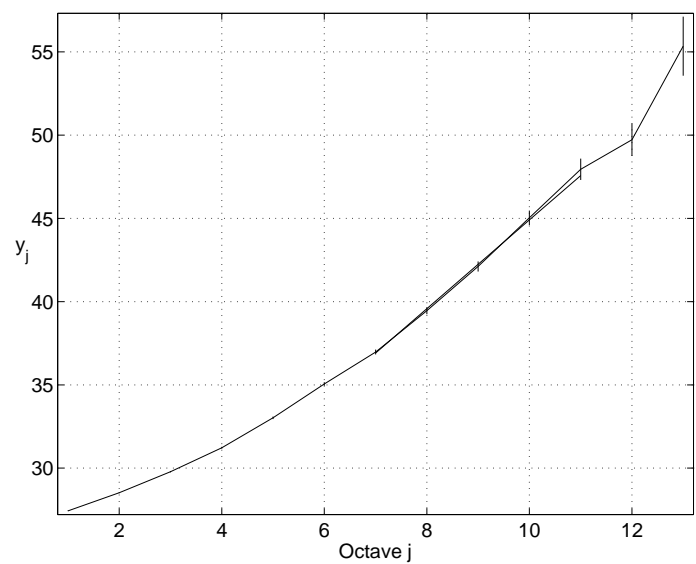

Fig. 4. LDestimate diagram of packets interarrival times (ms)

\section{REFERENCES}

[ABR98] P. Abry, and D. Veitch, Wavelet analysis of long range dependent traffic, IEEE Trans. Inform. Theory, vol. 4, no. 1, 1998.

[CLE00] J. Cleary, S. Donnely, I. Graham, A. McGregor and M. Pearson, Design principles for accurate passive measurement, PAM (Passive and Active Measurements) Workshop, Hamilton, New Zealand, April 2000.

[CRO97] M. Crovella and A. Bestavros, Self-similarity in World Wide Web traffic : Evidence and possible causes, IEEE/ACM Trans. on Networking, Vol. 5, n. 6, pp. 835-846, Dec. 1997.

[FAL96] Fall, K., Network emulation in the Vint/NS simulator, proceedings of ISCC'99, July 1999.

[PAR96] K. Park, G. Kim and M. Crovella, On the relationship between file sizes, transport protocols, and self-similar network traffic, Proc. of IEEE ICNP, 1996.

[PAX95] V. Paxson and S. Floyd, Wide-Area Traffic: The Failure of Poisson Modeling, IEEE/ACM Transactions on Networking, Vol. 3 No. 3, June 1995.

[PAX01] S. Floyd and V. Paxson, Difficulties in Simulating the Internet, IEEE/ACM Transactions on Networking, Vol.9, No.4, pp.392-403, August 2001.

[VER00] A. Veres, Zs. Kenesi, S. Molnar, G. Vattay, On the propagation of long-range dependence in the Internet, SIGCOMM'2000, Stockholm, Sweden, September 2000.

[ZHA01] Y. Zhang, N. Duffield, V. Paxson and S. Shenker, On the Constancy of Internet Path Properties, Proc. ACM SIGCOMM Internet Measurement Workshop (IMW'2001), San Francisco, California, USA, November 2001. 